\title{
The Maximal Length of 2-Path in Random Critical Graphs
}

\author{
Vonjy Rasendrahasina $\left(\mathbb{D},{ }^{1}\right.$ Vlady Ravelomanana, ${ }^{2}$ and Liva Aly Raonenantsoamihaja ${ }^{3}$ \\ ${ }^{1}$ ENS-Université d'Antananarivo, Antananarivo, Madagascar \\ ${ }^{2}$ IRIF UMR CNRS 8243, Université Denis Diderot, Paris, France \\ ${ }^{3}$ Faculté des Sciences, Université d'Antananarivo, Antananarivo, Madagascar
}

Correspondence should be addressed to Vonjy Rasendrahasina; rasendrahasina@gmail.com

Received 1 December 2017; Accepted 3 April 2018; Published 14 May 2018

Academic Editor: Bruno Carpentieri

Copyright (C) 2018 Vonjy Rasendrahasina et al. This is an open access article distributed under the Creative Commons Attribution License, which permits unrestricted use, distribution, and reproduction in any medium, provided the original work is properly cited.

Given a graph, its 2-core is the maximal subgraph of $G$ without vertices of degree 1. A 2-path in a connected graph is a simple path in its 2-core such that all vertices in the path have degree 2, except the endpoints which have degree $\geqslant 3$. Consider the Erdős-Rényi random graph $\mathbb{G}(n, M)$ built with $n$ vertices and $M$ edges uniformly randomly chosen from the set of $\left(\begin{array}{c}n \\ 2\end{array}\right)$ edges. Let $\xi_{n, M}$ be the maximum 2-path length of $\mathbb{G}(n, M)$. In this paper, we determine that there exists a constant $c(\lambda)$ such that $\mathbb{E}\left(\xi_{n,(n / 2)\left(1+\lambda n^{-1 / 3}\right)}\right) \sim$ $c(\lambda) n^{1 / 3}$, for any real $\lambda$. This parameter is studied through the use of generating functions and complex analysis.

\section{Preliminaries}

Let us recall that an undirected graph $G$ is a couple $(V, E)$, where $V$ is the set of vertices and $E$ the set of edges, and an edge is an unordered pair of vertices. If we allow an edge between a vertex and itself (loop) or multiple edges between two vertices, we obtain a multigraph. An undirected graph without loops or multiple edges is known as a simple graph. A path in graph $G=(V, E)$ is sequence of vertices $\left\langle v_{0}, v_{1}, \ldots, v_{k}\right\rangle$, where $\left\{v_{i}, v_{i+1}\right\} \in E$ for $i \in \llbracket 0, k-1 \rrbracket$ and $v_{i} \neq v_{j}$ for $i \neq j$ except that its first vertex $v_{0}$ might be the same as its last $v_{k}$. When any two vertices of $G$ are connected by a path $G$ is called connected.

A connected graph has excess if it has more edges than vertices. A connected component of excess $\ell$ is also called $\ell$-component. A tree or acyclic component is a connected component of excess -1 , an unicyclic component in a connected component of excess 0 . If $\ell \geqslant 1, \ell$-components are called complex. A graph (not necessarily connected) is called complex when all its components are complex. The total excessr of a graph is the number of edges plus the number of acyclic components, minus the number of vertices. In other words, the total excess of a graph is the sum of the excess of its complex components. Note that the total excess of a tree component is equal to 0 whereas its excess is equal to -1 and the total excess of a graph is nonnegative.

Given a graph $G$, its 2-core is obtained by deleting recursively all nodes of degree 1. A 3-core or kernel of a complex graph is the graph obtained from its 2-core by repeating the following process on any vertex of degree two: for a vertex of degree two, we can remove it and splice together the two edges that it formerly touched. We observe that $G$, its 2-core, and its kernel have the same excess. A graph is said cubic or 3-regular if all of its vertices are of degree 3. A graph is called clean if its 3-core is 3-regular (see [1]).

A random graph $\mathbb{G}(n, M=c n)$ is called critical if the density $c=1 / 2 \pm \mathcal{O}\left(n^{-1 / 3}\right)$. Such a graph contains a complex component with nonzero probability $[2,3]$. Janson et al. [1] proved these graphs are clean (its complex components are clean) with high probability when the size of graph goes to infinity.

Theorem 1. The maximum 2-path length $\xi_{n, M}$ of $\mathbb{G}(n, M)$ satisfies

$$
\mathbb{E}\left(\xi_{n,(n / 2)\left(1+\lambda n^{-1 / 3}\right)}\right) \sim c(\lambda) n^{1 / 3},
$$


where

$$
\begin{aligned}
c(\lambda) & =\frac{1}{\alpha} \int_{0}^{+\infty}(1 \\
& \left.-\sum_{r \geqslant 0} \sqrt{2 \pi} e_{r} A(3 r+1 / 2, \lambda)\left(1-e^{-x}\right)^{3 r}\right) d x,
\end{aligned}
$$

where $\alpha$ is the positive solution of

$$
\lambda=\alpha^{-1}-\alpha
$$

$e_{r}$ is given by

$$
e_{r}=\frac{(6 r) !}{2^{5 r} 3^{2 r}(3 r) !(2 r) !},
$$

and the function $A$ is defined by

$$
A(y, \lambda)=\frac{e^{-\lambda^{3} / 6}}{3^{(y+1) / 3}} \sum_{k \geqslant 0} \frac{\left((1 / 2) 3^{2 / 3} \lambda\right)^{k}}{k ! \Gamma((y+1-2 k) / 3)} .
$$

We remark that for Erdős-Rényi random graph $\mathbb{G}(n, p=$ $(1+\varepsilon) / n)$, Ding et al. [4] and Ding et al. [5] provided a complete characterisation of the structure of the giant component when $\varepsilon=o(1)$ but $\varepsilon^{3} n \rightarrow \infty$. Using our notation, $\varepsilon=\lambda n^{-1 / 3}$ but $\lambda \rightarrow \infty$ as $n \rightarrow \infty$. They describe that the $2-$ core of a graph is obtained by "stretching" the edges into paths of lengths i.i.d. geometric with mean $1 / \varepsilon=\lambda^{-1} n^{1 / 3}$. Next, in order to reconstruct the graph, they attached trees to vertices i.i.d. Poisson $(1-\varepsilon)$-Galton-Watson.

\section{Enumerative Tools}

As shown in $[1,3]$, exponential generating functions (EGFs) can lead to stringent results about the main characteristics of random graphs when they apply. Let us recall briefly the main EGFs involved in our proofs. We refer the reader to Harary and Palmer [6] for EGFs related to graphical enumeration.

For $n \geqslant 0$ and $-1 \leqslant \ell \leqslant\left(\begin{array}{l}n \\ 2\end{array}\right)$, let $c(n, n+\ell)$ be the number of connected graphs of excess $\ell$ and

$$
W_{\ell}(z)=\sum_{n=0}^{+\infty} c(n, n+\ell) \frac{z^{n}}{n !},
$$

the associated EGF. We know from [7] that $c(n, n-1)=n^{n-2}$ and

$$
W_{-1}(z)=\sum_{n=1}^{+\infty} n^{n-2} \frac{z^{n}}{n !}=T(z)^{2}-\frac{1}{2} T(z)^{2},
$$

where $T(z)$ is the EGF of rooted Cayley trees given by

$$
T(z)=z e^{T(z)}=\sum_{n=1}^{+\infty} n^{n-1} \frac{z^{n}}{n !} .
$$

We also have (see, e.g., [1, Equation (3.5)])

$$
W_{0}(z)=\frac{1}{2} \log \frac{1}{1-T(z)}-\frac{T(z)}{2}-\frac{T(z)^{2}}{4} .
$$

Wright [8] has shown that the EGFs $\left(W_{\ell}\right)_{\ell \geqslant 1}$ can be expressed in terms of $T(z)$. More precisely, Wright proved that for each $\ell \geqslant 1$ there exist rational coefficients $w_{\ell, d}, d \in\{0, \ldots, 3 \ell+2\}$ such that

$$
W_{\ell}(z)=\sum_{d=0}^{3 \ell+2} \frac{w_{\ell, d}}{(1-T(z))^{3 \ell-d}} .
$$

The coefficients $b_{\ell}:=w_{\ell, 0}$ are known as Wright's constants (see [9]). For complex graphs, denote by $E_{r}(z)$ the EGF of these graphs of excess $r$. Then we have $E_{0}(z)=1$ (empty graphs) and $E_{1}(z)=W_{1}(z)$. More generally, as detailed in $[1$, Section 8$]$, the $\operatorname{EGF} E_{r}(z)$ satisfies

$$
\sum_{r=0}^{+\infty} E_{r}(z)=\exp \left(\sum_{r=1}^{+\infty} W_{\ell}(z)\right) .
$$

Following [1], the EGF $E_{r}(z)$ can also be expressed as a rational function of $T(z)$

$$
E_{r}(z)=\sum_{d \geqslant 0} e_{r, d} \frac{T(z)^{5 r-d}}{(1-T(z))^{3 r-d}}=\sum_{d \geqslant 0} \frac{e_{r, d}^{\prime}}{(1-T(z))^{3 r-d}},
$$

where $e_{r}:=e_{r, 0}=e_{r, 0}^{\prime}$. The coefficients $\left(e_{r}\right)$ and $\left(b_{r}\right)$ are related by

$$
\begin{aligned}
e_{0} & =1, \\
r e_{r} & =r b_{r}+\sum_{j=1}^{r-1} j b_{j} e_{r-j} \quad \text { as } r \geqslant 1 .
\end{aligned}
$$

As shown in $[1,10]$, we remark that the dominant asymptotic behavior of $\left[z^{n}\right] W_{\ell}(z)$ and $\left[z^{n}\right] E_{r}(z)$ (for any power series $A(z)=\sum a_{n} z^{n},\left[z^{n}\right] A(z)$ denotes the $n$th coefficient of $A(z)$, namely, $\left[z^{n}\right] A(z)=a_{n}$.) is governed by the leading coefficients $b_{\ell}$ and $e_{r}$. In particular, if $\ell$ and $r$ are about $o\left(n^{1 / 3}\right)$, these EGFs satisfy

$$
\begin{aligned}
& W_{\ell}(z) \asymp_{\ell} \frac{b_{\ell}}{(1-T(z))^{3 \ell}}, \\
& E_{r}(z) \asymp_{r} \frac{e_{r}}{(1-T(z))^{3 r}},
\end{aligned}
$$

where $A(z) \asymp_{\ell} B(z)$ if and only if $\left[z^{n}\right] A(z) \sim\left[z^{n}\right] B(z)$ as $n \rightarrow$ $+\infty$ and $\ell=o\left(n^{1 / 3}\right)$.

The EGF $b_{\ell} /(1-T(z))^{3 \ell}$ (resp., $\left.e_{r} /(1-T(z))^{3 r}\right)$ can be interpreted as EGF of connected graphs (resp., complex graphs) whose kernels are 3-regular. Such a graph has exactly $3 \ell$ (resp., 3r) 2-paths. A 2-path in the 2-core is enumerated by $1 /(1-z)$. Substituting $z$ by $T(z)$ to obtain $1 /(1-T(z))$ means attaching tree to each vertex of the 2-core. Since a 3regular graph of excess $r$ has exactly $3 r$ edges, the associated graph has $3 r$ 2-paths. Note that, in the stated range, complex graphs and multigraphs of excess $r$ are both enumerated by $e_{r} /(1-T(z))^{3 r}$ and for large $r, e_{r} \sim b_{r}$ (see [1, Equation (7.16)] and $[9$, Section 7$])$.

In our case, we need to control the length of each 2-path to a graph. We restrict our attention to complex graphs whose 2paths are of length at most $k$. So, instead of allowing 2-path of 
any length $\left(1 /(1-T(z))=1+T(z)+T(z)^{2}+\cdots+T(z)^{k}+\cdots\right)$, we use a 2-path of length at most $k\left(1+T(z)+T(z)^{2}+\cdots+T(z)^{k}\right)$. The associated EGF is

$$
\begin{aligned}
e_{r}\left(1+T(z)+T(z)^{2}+\cdots+T(z)^{k}\right)^{3 r} \\
\quad=e_{r} \frac{\left(1-T(z)^{k+1}\right)^{3 r}}{(1-T(z))^{3 r}} .
\end{aligned}
$$

\section{Proof of Theorem 1}

Consider a graph with $n$ vertices, $M$ edges, and a total excess $r$. Such a graph contains exactly $n-M+r$ tree components. They are enumerated by the following EGF:

$$
\frac{W_{-1}(z)^{n-M+r}}{(n-M+r) !} \exp \left(W_{0}(z)\right) E_{r}(z) .
$$

Since the total number of graphs with $n$ vertices and $M$ edges is $\left(\begin{array}{c}n \\ 2 \\ M\end{array}\right)$, the probability that a random $(n, M)$-graph (graphs with $n$ vertices and $M$ edges) is of total excess $r$ is

$$
\frac{n !}{\left(\begin{array}{c}
n \\
2 \\
M
\end{array}\right)}\left[z^{n}\right] \frac{W_{-1}(z)^{n-M+r}}{(n-M+r) !} \exp \left(W_{0}(z)\right) E_{r}(z) .
$$

Similarly, the probability that a random $(n, M)$-graph is of total excess $r$ and has no 2-path of length greater than $k$ is

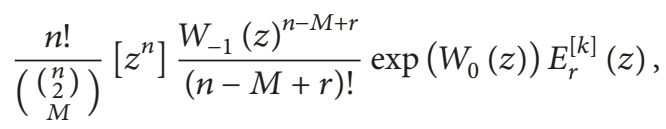

where $E_{r}^{[k]}(z)$ denotes the EGF of all complex components of total excess $r$ whose 2-paths are of length at most $k, k \geqslant$ 1 . Then summing over $r$, we get that the probability that a random $(n, M)$-graph has no 2-path of length greater than $k$ is

$$
\begin{aligned}
& \mathbb{P}\left(\xi_{n, M} \leqslant k\right)
\end{aligned}
$$

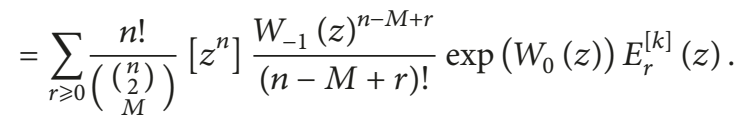

Following discussion in the previous section and using (15), the probability of a random $\mathbb{P}\left(\xi_{n, M} \leqslant k\right)$ is asymptotically equivalent to

$$
\begin{aligned}
\mathbb{P}\left(\xi_{n, M} \leqslant k\right) \sim & \sum_{r \geqslant 0} \frac{n !}{\left.\left(\begin{array}{c}
n \\
2
\end{array}\right)\right)}\left[z^{n}\right] \frac{W_{-1}(z)^{n-M+r}}{(n-M+r) !} \\
& \cdot \exp \left(W_{0}(z)\right) \frac{e_{r}\left(1-T(z)^{k+1}\right)^{3 r}}{(1-T(z))^{3 r}} .
\end{aligned}
$$

As in [11, Section 4] where Flajolet et al. described generating functions based methods to study extremal statistics on random mappings, we characterize the expectation of $\xi_{n, M}$ by means of truncated generating functions aforementioned. In fact, the mean value of $\xi_{n, M}$ is given by

$$
\mathbb{E}\left(\xi_{n, M}\right)=\sum_{k \geqslant 0} k \mathbb{P}\left[\xi_{n, M}=k\right]=\sum_{k \geqslant 0}(1-\mathbb{P}[X \leqslant k]) .
$$

Then, combining (19), (20), and (21), we have

$$
\begin{aligned}
& \mathbb{E}\left(\xi_{n, M}\right) \sim \sum_{k \geqslant 0}\left(1-\sum_{r \geqslant 0} \frac{n !}{\left(\begin{array}{c}
n \\
2 \\
M
\end{array}\right)}\left[\begin{array}{l}
n \\
\text { n }
\end{array}\right] \frac{W_{-1}(z)^{n-M+r}}{(n-M+r) !}\right. \\
& \left.\cdot \exp \left(W_{0}(z)\right) \frac{e_{r}\left(1-T(z)^{k+1}\right)^{3 r}}{(1-T(z))^{3 r}}\right) .
\end{aligned}
$$

To compute $E\left(\xi_{n, M}\right)$, we use the following lemma.

Lemma 2. Let $M=(n / 2)\left(1+\lambda n^{-1 / 3}\right)$. For any natural integers $r$ and $k$, one has

$$
\begin{gathered}
p_{r, k}(n, M):=\frac{n !}{\left.\left(\begin{array}{c}
n \\
2
\end{array}\right)\right)}\left[z^{n}\right] \frac{W_{-1}(z)^{n-M+r}}{(n-M+r) !} \exp \left(W_{0}(z)\right) \\
\cdot \frac{\left(1-T(z)^{k}\right)^{3 r}}{(1-T(z))^{3 r}}=\sqrt{2 \pi} A\left(3 r+\frac{1}{2}, \lambda\right) \\
\cdot\left(1-e^{k \alpha n^{-1 / 3}}\right)^{3 r}\left(1+\mathcal{O}\left(\frac{\lambda^{4}}{n^{1 / 3}}\right)\right) .
\end{gathered}
$$

Proof. We set

$$
\begin{aligned}
& \text { St }(n, M, r)=\frac{n !}{\left(\begin{array}{c}
n \\
2
\end{array}\right)(n-M+r) !}, \\
& \mathrm{Ca}(n, M, r) \\
& \quad=\left[z^{n}\right] W_{-1}(z)^{n-M+r} e^{W_{0}(z)} \frac{\left(1-T(z)^{k}\right)^{3 r}}{(1-T(z))^{3 r}} .
\end{aligned}
$$

First, using Stirling's formula, we obtain

$$
\begin{aligned}
\operatorname{St}(M, n)= & \sqrt{2 \pi n} \frac{2^{n-M+r}}{n^{r}} \exp \left(-\frac{\lambda^{3}}{6}+\frac{3}{4}-n\right) \\
& \cdot\left(1+O\left(\frac{\lambda^{4}}{n^{1 / 3}}\right)\right) .
\end{aligned}
$$

Next, using Cauchy integral's formula and substituting $z$ by $z e^{-z}$, we obtain

$$
\begin{aligned}
& \mathrm{Ca}(M, n)=\frac{1}{2 \pi i} \oint\left(T(z)-\frac{T(z)^{2}}{2}\right)^{n-M+r} \\
& \cdot e^{-T(z) / 2-T(z)^{2} / 4} \frac{\left(1-T(z)^{k}\right)^{3 r}}{(1-T(z))^{3 r+1 / 2}} \frac{d z}{z^{n+1}}=\frac{2^{M-n-r} e^{n}}{2 \pi i} \\
& \cdot \oint g(z) \exp (n h(z)) \frac{d z}{z}
\end{aligned}
$$


where

$$
\begin{aligned}
& g(z)=\frac{\left(2 z-z^{2}\right)^{r} e^{-z / 2-z^{2} / 4}\left(1-z^{k}\right)^{3 r}}{(1-z)^{3 r-1 / 2}}, \\
& h(z)=z-1-\log z-\left(1-\frac{M}{n}\right) \log \frac{1}{1-(z-1)^{2}} .
\end{aligned}
$$

The contour in (26) should keep $|z|<1$. At the critical value $M=n / 2$, we also have $h(1)=h^{\prime}(1)=h^{\prime \prime}(1)=0$. This triple zero occurs in the procedure Janson et al. [1] used when investigating the value of the integral for large $n$. Let $v=n^{-1 / 3}$, and let $\alpha$ be the positive solution of (3). Following the proof of [1, Lemma 3], we will evaluate (26) on the path $z=e^{-(\alpha+i t) \nu}$, where $t$ runs from $-\pi n^{1 / 3}$ to $\pi n^{1 / 3}$ :

$$
\oint f(z) \frac{d z}{z}=i v \int_{-\pi n^{1 / 3}}^{\pi n^{1 / 3}} f\left(e^{-(\alpha+i t) v}\right) d t .
$$

The main contribution to the value of this integral comes from the vicinity of $t=0$. The magnitude of $e^{h(z)}$ depends on the real part of $h(z)$, namely, $\operatorname{Re} h(z)$. $\operatorname{Re} h\left(e^{-(\alpha+i t) \nu}\right)$ decreases as $|t|$ increases and $\left|e^{n h(z)}\right|$ has its maximum on the circle $z=$ $e^{-(\alpha+i t) v}$ when $t=0$.

We have for $n h\left(e^{-s v}\right)$

$$
n h\left(e^{-s v}\right)=\frac{1}{3} s^{3}+\frac{1}{2} \lambda s^{2}+O\left(\left(\lambda^{2} s^{2}+s^{4}\right) \nu\right),
$$

uniformly in any region such that $|s v|<\log 2$. In $[1$, equation (10.7)], the authors define

$$
A(y, \mu)=\frac{1}{2 \pi i} \int_{\Pi(1)} s^{1-y} e^{K(\mu, s)} d s,
$$

where $K(\mu, s)$ is the polynomial

$$
K(\mu, s)=\frac{(s+\mu)^{2}(2 s-\mu)}{6}=\frac{s^{3}}{3}+\frac{\mu s^{2}}{2}-\frac{\mu^{3}}{6}
$$

and $\Pi(\alpha)$ is a path in the complex plane that consists of the following three straight line segments:

$$
s(t)= \begin{cases}-e^{-\pi i / 3} t, & \text { for }-\infty<t \leqslant-2 \alpha ; \\ \alpha+i t \sin \frac{\pi}{3}, & \text { for }-2 \alpha \leqslant t \leqslant+2 \alpha ; \\ e^{+\pi i / 3} t, & \text { for }+2 \alpha \leqslant t<+\infty .\end{cases}
$$
as (5).

In particular, they proved that $A(y, \mu)$ can be expressed

For the function $g(z)$, we have

$$
\begin{aligned}
g\left(e^{-s v}\right) & =\frac{\left(2 e^{-s v}-e^{-2 s v}\right)^{r}}{\left(1-e^{-s v}\right)^{3 r-1 / 2}} e^{-e^{-s v} / 2-e^{-2 s v} / 4}\left(1-e^{-k s v}\right)^{3 r} \\
& =(s v)^{1 / 2-3 r} e^{-3 / 4}\left(1-e^{-k s v}\right)^{3 r}(1+O(s \nu)) .
\end{aligned}
$$

For $g(z) e^{n h(z)}$ in the integrand of (26), we have

$$
\begin{aligned}
& e^{-\lambda^{3} / 6} f\left(e^{-s v}\right)=e^{-3 / 4} v^{1 / 2-3 r}\left(1-e^{-k s v}\right)^{3 r} \\
& \cdot s^{1-(3 r+1 / 2)} e^{K(\lambda, s)}\left(1+O(s v)+O\left(\lambda^{2} s^{2} v\right)\right. \\
& \left.+O\left(s^{4} \nu\right)\right)
\end{aligned}
$$

when $s=O\left(n^{1 / 12}\right)$. Next,

$$
\begin{aligned}
& \frac{e^{-\lambda^{3} / 6}}{2 \pi i} \oint g(z) e^{n h(z)} \frac{d z}{z} \\
& =e^{-3 / 4}\left(1-e^{-k \alpha \nu}\right)^{3 r} \nu^{3 / 2-3 r} A\left(3 r+\frac{1}{2}, \lambda\right) \\
& \quad+O\left(\nu^{5 / 2-3 r} e^{-\lambda^{3} / 6} \lambda^{3 r / 2+1 / 4}\right),
\end{aligned}
$$

where the error term has been derived from those already in [1]. The proof of the lemma is completed by multiplying (25), (26), and $e_{r}$.

Now, to complete the proof of the theorem, we use first Lemma 2 to get

$$
\begin{aligned}
& \mathbb{P}\left(\xi_{n, M} \leqslant k\right) \\
& \quad \sim \sum_{r \geqslant 0} \sqrt{2 \pi} e_{r} A\left(3 r+\frac{1}{2}, \lambda\right)\left(1-e^{-(k+1) \alpha n^{-1 / 3}}\right)^{3 r} .
\end{aligned}
$$

Next, using Euler-Maclaurin summation, and after a change of variable $\left(x=(k+1) \alpha n^{-1 / 3}\right.$ so $d x / d k=\alpha n^{-1 / 3}$ and $\left.d k=\alpha^{-1} n^{1 / 3} d x\right)$, we get

$$
\begin{aligned}
& \mathbb{E}\left(\xi_{n, M}\right)=\sum_{k=0}^{+\infty}\left(1-\mathbb{P}\left(\xi_{n, M} \leqslant k\right)\right), \\
& \sim \sum_{k=0}^{+\infty}(1 \\
& \left.\quad-\sum_{r \geqslant 0} \sqrt{2 \pi} e_{r} A\left(3 r+\frac{1}{2}, \lambda\right)\left(1-e^{-(k+1) \alpha n^{-1 / 3}}\right)^{3 r}\right) \\
& \sim \int_{0}^{+\infty}(1 \\
& \left.\quad-\sum_{r \geqslant 0} \sqrt{2 \pi} e_{r} A\left(3 r+\frac{1}{2}, \lambda\right)\left(1-e^{-x}\right)^{3 r}\right) d k \\
& \sim \alpha^{-1} n^{1 / 3} \int_{0}^{+\infty}(1 \\
& \left.\quad-\sum_{r \geqslant 0} \sqrt{2 \pi} e_{r} A\left(3 r+\frac{1}{2}, \lambda\right)\left(1-e^{-x}\right)^{3 r}\right) d x .
\end{aligned}
$$

\section{Conclusion}

In this paper, we have studied the expectation of the maximal length of 2-path in random critical graph by means of 
enumerative and analytic combinatorics approaches when the size of the graph goes to infinity. Our analysis gives a precise description of the parameter near the critical point.

\section{Conflicts of Interest}

The authors declare that they have no conflicts of interest.

\section{Acknowledgments}

This work was supported by the French project ANR project MetACOnc, ANR-15-CE40-0014.

\section{References}

[1] S. Janson, D. E. Knuth, T. Luczak, and B. Pittel, "The birth of the giant component," Random Structures \& Algorithms, vol. 4, no. 3, pp. 231-358, 1993.

[2] P. Erdős and A. Rényi, "On the evolution of random graphs," Publications of the Mathematical Institute of the Hungarian Academy of Sciences, vol. 5, pp. 17-61, 1960.

[3] P. Flajolet, D. E. Knuth, and B. Pittel, "The first cycles in an evolving graph," Discrete Mathematics, vol. 75, no. 1-3, pp. 167215, 1989.

[4] J. Ding, E. Lubetzky, and Y. Peres, "Anatomy of the giant component: the strictly supercritical regime," European Journal of Combinatorics, vol. 35, pp. 155-168, 2014.

[5] J. Ding, J. H. Kim, E. Lubetzky, and Y. Peres, "Anatomy of a young giant component in the random graph," Random Structures \& Algorithms, vol. 39, no. 2, pp. 139-178, 2011.

[6] F. Harary and E. M. Palmer, Graphical enumeration, Academic Press, 1973.

[7] A. Cayley, "A theorem on trees," The Quarterly journal of pure and applied mathematics, vol. 23, pp. 376-378, 1889.

[8] E. M. Wright, "The number of connected sparsely edged graphs," Journal of Graph Theory, vol. 1, no. 4, pp. 317-330, 1977.

[9] S. Janson, "Brownian excursion area, Wright's constants in graph enumeration, and other Brownian areas," Probability Surveys, vol. 4, pp. 80-145, 2007.

[10] E. M. Wright, "The number of connected sparsely edged graphs III. Asymptotic results," Journal of Graph Theory, vol. 4, no. 4, pp. 393-407, 1980.

[11] P. Flajolet, M. Andrew, and Odlyzko, "Random mapping statistics," in Proceedings of the Advances in Cryptology - EUROCRYPT '89, Workshop on the Theory and Application of of Cryptographic Techniques, pp. 329-354, Houthalen, Belgium, 1989. 


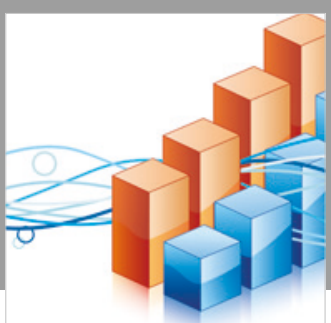

Advances in

Operations Research

\section{-n-m}
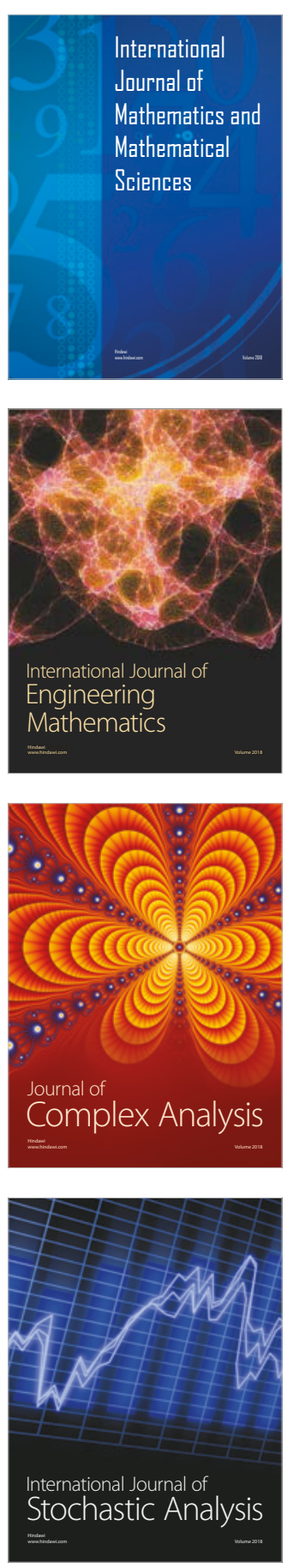
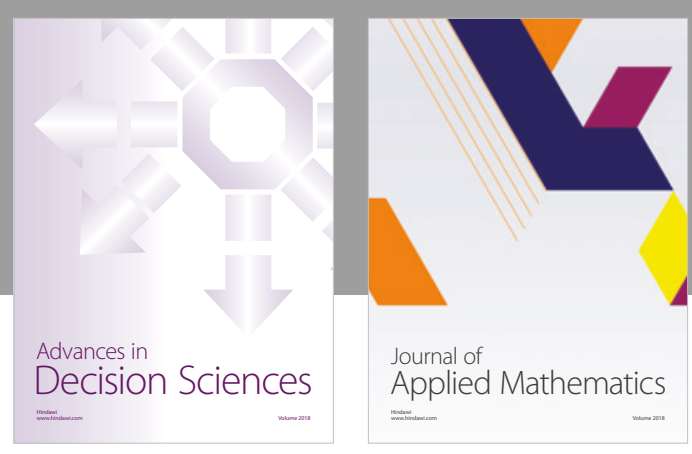

Journal of

Applied Mathematics
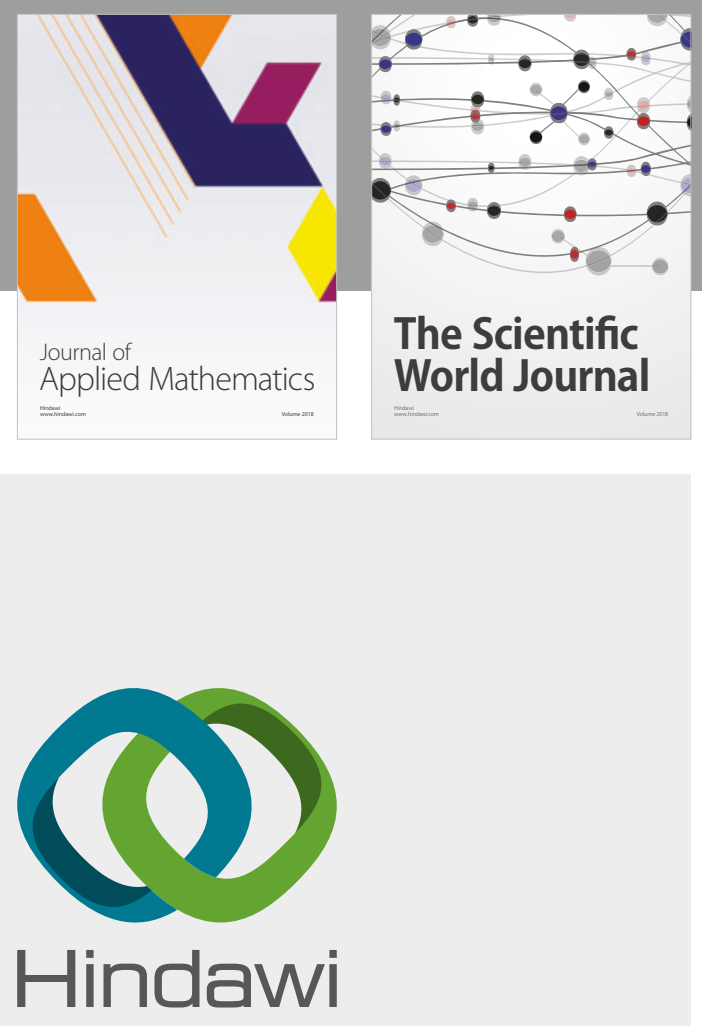

Submit your manuscripts at

www.hindawi.com

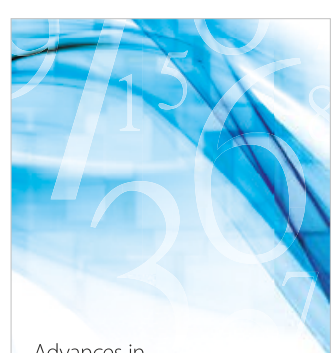

Advances in
Numerical Analysis
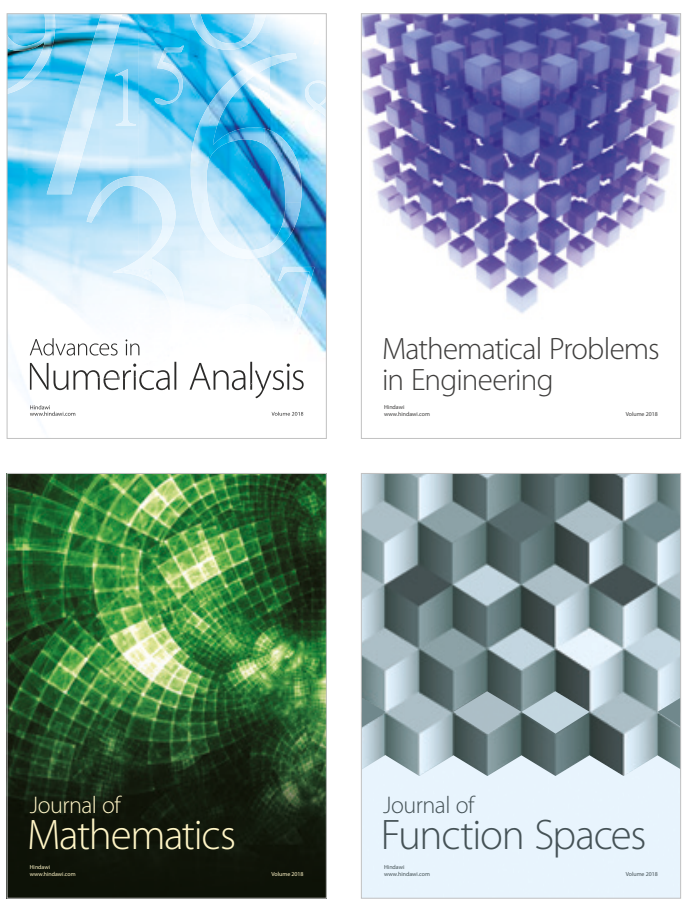

Mathematical Problems in Engineering

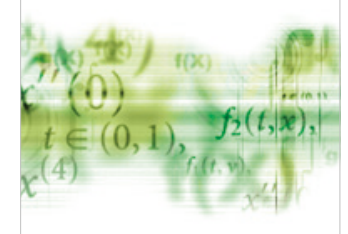

International Journal of

Differential Equations

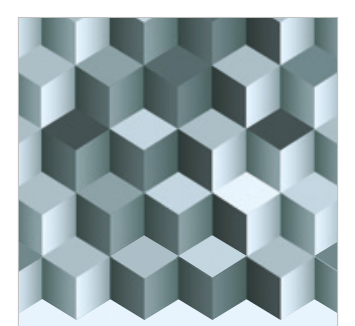

Journal of

Function Spaces

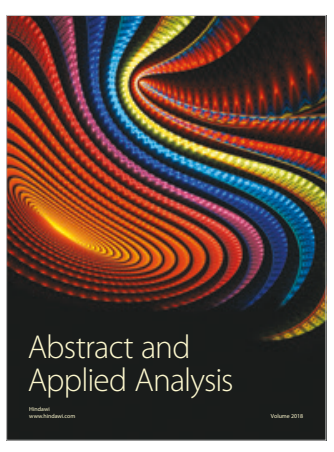

The Scientific

World Journal

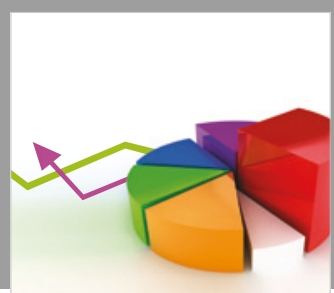

Journal of

Probability and Statistics
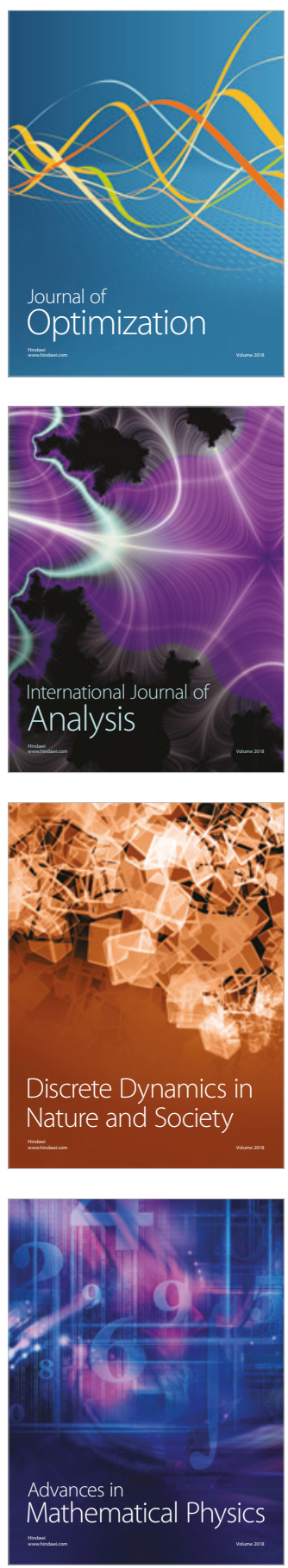